\title{
THE DEVELOPMENT OF THE FAULTS ACCOMMODATION SYSTEM FOR ACTUATORS OF MULTILINK MANIPULATORS
}

\author{
FILARETOV, V[ladimir] F[edorovich]; ZHIRABOK, A[lexey] N[il]; \\ ZUEV, A[lexander] \& PROTCENKO, A[leksandr]
}

\begin{abstract}
In this paper, a method for the synthesis of an effective faults accommodation system for actuators of multilink manipulators which are described by nonlinear differential equations with variable parameters is proposed. This system ensures insensitivity to faults appearing in actuators due to specifically formed additional control signal. This allows ensuring the efficiency of operating of manipulators in aggressive and extremal environment. The results of simulation confirmed the efficiency of the proposed method.
\end{abstract}

Keywords: Diagnosis, faults accommodationl, actuators, multilink manipulator

\section{INTRODUCTION}

Today the area of use of multilink manipulators (MM) has considerably extended and also the operations which are carried out by them are become more complicated. At that, the reliability and safety of this complex equipment at operating in standalone mode have the particular importance. In order to improve these factors, it's necessary to provide quality diagnosis of the main units and subsystems of MM due to the timely detecting and eliminating the faults before the appearance of emergency situations. An important task, which leads to quality improvements and efficiency of $\mathrm{MM}$ in case of arising of faults is the formation of special control signals, allowing to automatically save (stabilize) the most important characteristics of these devices during the time of their operation. The solution to this problem is called synthesis of fault tolerance control or faults accommodation [1]. The accommodation process consists of two main steps: 1. diagnosis of faults namely detection, localization and determination of their value; 2. tuning of control systems of MM to eliminate the acting of these faults.

Usually the diagnosis of faults in the actuators of MM are carry out by means of diagnostic observers. There are several methods for synthesis of diagnostic observers for nonlinear dynamic objects [2-5], which are quite difficult to implement because they require intensive calculations and assume differentiability of the functions included in the model of objects. This complicates the detection, localization and identification of faults in actuators of multilink manipulators which are described by complex nonlinear differential equations with variable parameters that take into account the interactions between all degrees of freedom of manipulators, dry friction and etc.

One of the main problems, solved at synthesis of diagnostic observers, is to provide insensitivity of process of diagnosing to possible significant mismatch between the initial states of the diagnosed system and the observers. This is achieved, usually by the introduction of feedback by signals of residuals in the observers [1-3].

At the present time there are several approaches to the synthesis of faults accommodation systems for multilink manipulators, which allow to on-line compensating the effects from faults. Generally these approaches are based on methods of $\mathrm{H}_{\infty}$-optimization [6,7], tracking the reference model [8] and adaptive control [9]. In the paper[10] the method is proposed for forming a control signal, which ensures complete independence from the effects caused by the defects, without the procedure of estimating their value.

The conducted analysis showed that due to the high complexity of realization of control signals or low performance, most of these methods and approaches cannot be efficiently used at the synthesis of the faults accommodation system for MM. Thus, at present the task of developing a new, easily realizable and effective synthesis method of fault tolerance control for multilink manipulators, whose dynamics is described by complex nonlinear differential equations with variable parameters, still remains unsolved.

\section{TASK SETTING AND DEFINITION OF EFFECTIVE WAYS OF ITS DECISION}

Let us to consider MM with $n$ degrees of freedom which is driven by $n$ actuators with DC motors. Each actuator has sensors of the position, speed of rotation, current, regulator $R$ that provides the specified quality indicators and adaptive regulator that compensates the interactions between the degrees of mobility of the manipulator. It is assumed that the following types of defects are possible in the actuators of MM: $d_{1}-$ fault caused by errors in the sensors, which measure the position of output shaft of gear; $d_{2}$ - fault caused by an increase of value moment of dry friction $M_{d f 0}$ in the actuators; $d_{3}$ - fault caused by the change of active resistance of rotor circuit of DC motors (for example, when significant changing of temperature). The presence of these defects significantly reduces the quality of the actuators and accuracy of performance of specific technological operations.

In the presence of these faults, the each actuator of MM without feedback can be described by the differential equation in a matrix form as follows:

$$
\begin{gathered}
\dot{x}(t)=A x(t)+B u(t)+F(t)+E d(t), \\
\text { where } A=\left[\begin{array}{ccc}
0 & 1 / \mu & 0 \\
0 & -\left(k_{v}+h\right) /(J+H) & k_{m} /(J+H) \\
0 & -k_{\omega} / L & -R / L
\end{array}\right] ; x=\left[\begin{array}{c}
\alpha_{l} \\
\dot{\alpha} \\
I
\end{array}\right] ;
\end{gathered}
$$


$B=\left[\begin{array}{ccc}0 & 0 & 0 \\ 0 & -M_{E} /(J+H) & 0 \\ 0 & 0 & k_{a} / L\end{array}\right] ; u=\left[\begin{array}{l}0 \\ 1 \\ u\end{array}\right] ; G=\left[\begin{array}{l}0 \\ 1 \\ 0\end{array}\right]^{\mathrm{T}} ; d=\left[\begin{array}{l}d_{1} \\ d_{2} \\ d_{3}\end{array}\right]$, $F=\tilde{F} \operatorname{sign}(G x) ; \tilde{F}=\left[\begin{array}{c}0 \\ -M_{d f 0} /(J+H) \\ 0\end{array}\right] ; E=\left[\begin{array}{lll}1 & 0 & 0 \\ 0 & 1 & 0 \\ 0 & 0 & 1\end{array}\right] ;$

$R, L, I$ are resistance, inductance and current of DC motor rotor circuits, accordingly; $k_{\omega}$ is a counter-EMF coefficient; $k_{a}$ is an amplifier coefficients; $k_{m}$ is a moment coefficient; $J$ is a moment of inertia of DC motor shaft and rotating parts of reducers; $\alpha_{l}$ is a turn angle of electric motor shaft; $\dot{\alpha}$ is a rotor speed; $\mu$ is a reducing ratio of the reducer; $h, H, M_{E}$ are the variable components of torque influences on actuators, including the interactions between all the degrees of freedom of the manipulator; $u$ is a input voltage of amplifiers.

At the operation of MM, each fault should be timely detected, and its influence on the quality of the work of MM should be eliminated. Obvious that significant variability of the parameters $h, H, M_{E}$, and the presence of the nonlinearity $F$ makes impossible the solution the task of synthesis of faults accommodation system for actuators of $\mathrm{MM}$ with traditional methods, focused on linear models of control objects.

Thus, in this paper the problem of creating an effective synthesis method of faults accommodation system for actuators with DC motors of multilink manipulators is formulated and solved. This method should take into account that actuators of $\mathrm{MM}$ are described by complex nonlinear differential equations with variable parameters.

To solve this problem, we propose a new synthesis method of faults accommodation system, which includes three main stages: 1) the synthesis of observers based on of logical-dynamic approach [11], which allows to solve the problem of diagnosing MM's actuators with nondifferentiable non-linearity by linear methods; 2) determination of the magnitudes of faults in the presence of large disturbances and the initial mismatch between states of MM's actuators and related observers; 3) the formation of control signals due to the use of additional signals of residuals which guarantee quick parrying of occurring faults and provides accurate stabilization of the dynamic properties of actuators.

This method can be used effectively at the development of accommodation systems to faults arising in DC motors of manipulators with any type of kinematic scheme.

\section{SYNTHESIS OF DIAGNOSTIC OBSERVERS}

The problem of detection and localization of faults in actuators with DC motors of multilink manipulators described by nonlinear differential equations with variable parameters can be effectively solved on the basis of logic-dynamic approach, which is very easy to implement because it uses linear methods and can work with nondifferentiable nonlinearities.

This approach includes three steps [11]. At the first stage, original nonlinear model of the actuators (1) is converted to a linear model by removing the nonlinear parts.
At the second stage, a bank of linear diagnostic observers is designed to detect and localize defects $d_{1}, d_{2}$ and $d_{3}$ in DC motors. Each observer is described by equation:

$$
\dot{x}_{*}(t)=A_{*} x_{*}(t)+B_{*} u(t)+N x(t),
$$

where $N \in R^{3}$, and symbol "*" marks the matrices and vectors, which describe the considered observer in contrast to the relevant matrices and vectors of actuator's model (1).

The residual $r(t)$ allowing to make decision about the presence or absence of faults in diagnosed system is formed as:

$$
r(t)=\Phi x(t)-x_{*}(t)
$$

where $\Phi \in R^{3}$ is a vector, which will be defined later.

For the synthesis of specific observers that are sensitive to defects $d_{1}, d_{2}$ and $d_{3}$, occuring in actuators of $\mathrm{MM}$, it is necessary to determine the matrices $A_{*}, B_{*}$ and vector $N$, describing the observer (2), and vector $\Phi$.

In the absence of faults, the following equation is performed after the end of transition process in observers: $\Phi x(t)=x_{*}(t)$.

Using the condition of the sensitivity of the residual (3) to the defect, which has the form [11]: $\Phi\left[\begin{array}{l}d_{1} \\ d_{2} \\ d_{3}\end{array}\right] \neq 0$, vectors $\Phi$ can be formed for each observers: for the observer $\mathrm{O}_{1}$, sensitive to the fault $d_{1}-\Phi_{1}=\left[\begin{array}{lll}1 & 0 & 0\end{array}\right]$; for

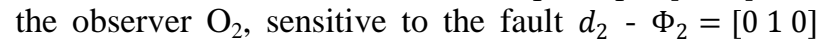
and for the observer $\mathrm{O}_{3}$, sensitive to the fault $d_{3}-$ $\Phi_{3}=\left[\begin{array}{lll}0 & 0 & 1\end{array}\right]$.

It is known [1-3] that the synthesis of linear observers is desirable in the Kronecker's canonical form. In this case, considering dimensions of the synthesized observers (2) we have $A_{*}=0$. With this, and also taking into account the following equalities [11]

$$
N=\Phi A, B_{*}=\Phi B,
$$

for the observer $\mathrm{O}_{1}$, sensitive to the fault $d_{1}$ and invariant

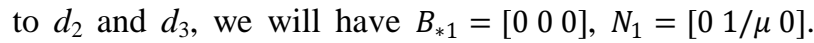
With this observer $\mathrm{O}_{1}$ will be described by the equations:

$$
\dot{x}_{* 1}(t)=\dot{\alpha} / \mu, \quad r_{1}(t)=\alpha_{l}-x_{* 1}
$$

For the observer $\mathrm{O}_{2}$, sensitive to the fault $d_{2}$ and invariant to $d_{1}$ and $d_{3}, \quad B_{* 2}=\left[\begin{array}{lll}0-\frac{M_{E}}{(J+H)} & 0\end{array}\right]$, $N_{2}=\left[0-\frac{k_{v}+h}{J+H} \frac{k_{m}}{(J+H)}\right]$. It is described by the equations:

$$
\begin{gathered}
\dot{x}_{* 2}(t)=-\frac{k_{v}+h}{J+H} \dot{\alpha}+\frac{k_{m}}{(J+H)} I-\frac{M_{E}}{(J+H)}, \\
r_{2}(t)=\dot{\alpha}-x_{* 2} .
\end{gathered}
$$

For the observer $\mathrm{O}_{3}$, sensitive to the defect $d_{3}$ and invariant to $d_{1}$ and $d_{2}: \quad B_{* 3}=\left[\begin{array}{lll}0 & 0 & k_{a} / L\end{array}\right]$, $N_{2}=\left[0\left(k_{v}+h\right) / L-R / L\right]$. It is described by the equations: 


$$
\begin{gathered}
\dot{x}_{* 3}(t)=\frac{k_{a}}{L} u-\frac{\left(k_{v}+h\right)}{L} \dot{\alpha}-\frac{R}{L} I, \\
r_{2}(t)=I-x_{* 3} .
\end{gathered}
$$

In the third stage of logic-dynamic approach the transition from linear to nonlinear observers is carried out. Formally, this procedure is carried out by adding nonlinear term $F$ to models of observers (5) - (7). In view of equalities $\Phi_{1} F=0, \Phi_{2} F=-\frac{M_{d f 0}}{J+H} \operatorname{sign}(G x)$ and $\Phi_{3} F=$ 0 , nonlinear component is added only in the second observer (6), the equation of which transformed to:

$$
\begin{gathered}
\dot{x}_{* 2}(t)=-\frac{k_{v}+h}{J+H} \dot{\alpha}+\frac{k_{m}}{(J+H)} I-\frac{M_{E}}{(J+H)}- \\
-\frac{M_{d f 0}}{J+H} \operatorname{sign}(\dot{\alpha}), \\
r_{2}(t)=\dot{\alpha}-x_{* 2} .
\end{gathered}
$$

Next, it will be shown that the synthesized observers (5), (7) and (8) allow to provide quality diagnostic of MM's actuators in real-time (1) detecting and localizing the arising faults.

\section{IDENTIFICATION OF VALUE OF THE DEFECTS}

After the detection of faults that occur in actuators of MM it's necessary to accurately determine the magnitude of these defects in real time, taking into account a possible mismatch of the initial states of the model (1) of actuators and synthesized observers (5), (7) (8). To ensure the sustainability of the diagnosis in the presence of initial mismatch of observers, we introduce a special feedback signal from the residual (3). The system of equations, describing each nonlinear observer with feedback, given that $A_{*}=0$, has the form:

$$
\dot{x}_{*}(t)=B_{*} u(t)+N x(t)+F^{*}+w r(t)
$$

where $F^{*}=\Phi F, w$ is a gain of feedback.

Then from (1), (4) and (9) follows that:

$$
\dot{r}(t)=\Phi \dot{x}(t)-\dot{x}_{*}(t)=w r(t)+d
$$

Equation (10) describes the behavior of the residuals in case of presence of faults, after the introduction of feedback loop in the observers. The solution of equation (10) has the form:

$$
r(t)=C_{1} e^{-w t}+d / w
$$

It's follows from expression (11) that the coefficient of the feedback determines the time of identification of fault size. The conducted research has shown at the synthesis of fault tolerance control systems for actuators of $\mathrm{MM}$ is desirable to choose $w=3 / T_{c}$, where $T_{c}$ is a time at which residual signal reaches $5 \%$ closeness of value $d / w$.

The relation between residuals and faults in actuators at the steady state is defined in the form: $r_{1}=d_{1} / w_{1}$, $r_{2}=d_{2} / w_{2}, r_{3}=d_{3} / w_{3}$.

With this in mind, as well as the fact, that $d_{1}=\dot{\tilde{\alpha}}_{l}$, $d_{2}=-\frac{\widetilde{M}_{d f 0}}{(J+H)} \operatorname{sign}(\dot{\alpha}), \quad d_{3}=-\frac{\widetilde{R}}{L} I, \quad$ the changes of parameters of actuators caused by faults, can be defined in following form:

$$
\begin{gathered}
\tilde{\alpha}_{l}=w_{1} \int r_{1} d t, \tilde{M}_{d f 0}=-r_{2} w_{2}(J+H) \operatorname{sign}(\dot{\alpha}), \\
\tilde{R}=-r_{3} L w_{3} / I .
\end{gathered}
$$

Thus introducing into each synthesized observer the feedback by residual signal with gain $w$, it is possible to ensure the sustainability of the process of identification and accurate determination of values of faults.

\section{SYNTHESIS OF SYSTEM OF ACCOMMODATION TO FAULTS}

After the identification of occurring faults it's necessary to solve the problem of accurate compensation by using the approaches described in $[1,3,10]$. But for the actuators of $\mathrm{MM}$ with significantly variable parameters, it is advisable to solve this problem by using method of synthesis of corrective devices that stabilize these parameters to the nominal level [12].

In the beginning of this method for actuators of multilink manipulators, the required dynamic properties are defined. These properties for DC motors are determined by the differential equation with constant coefficients, such as:

$$
L J \dddot{\alpha}+R J \ddot{\alpha}+k_{\omega} k_{m} \dot{\alpha}=k_{a} k_{m} \tilde{u},
$$

where $\tilde{u}$ is a control signal of input of adaptive regulator.

The expression for the highest derivative of the output variable $(\dddot{\alpha})$ is determined from this equation. Substitution of it into the original system of differential equation (1) with variable coefficients allows to obtain required control law as a function of lower derivatives of the output variable. This provides the specified dynamic properties and the quality of the control object. It is possible to stabilize the characteristics of each actuator at nominal level, by introducing the additional residuals formed by using observers (9) in control law. Finally fault tolerance control law by each actuator of MM takes the form:

$$
\begin{gathered}
u=\frac{J+H}{J} F(p, \varepsilon)\left[\alpha_{l}^{d}-\alpha_{l}-\tilde{\alpha}_{l}\right]+\frac{L\left(2 h+k_{v}\right)}{k_{a} k_{m}} \ddot{\alpha}+ \\
+\frac{1}{k_{a} k_{m}}\left[L \dot{h}+(R+\tilde{R})\left(h+k_{v}\right)+\right. \\
\left.+k_{\omega} k_{m}\left(1-\frac{J+H}{J}\right)\right] \dot{\alpha}+\frac{(R+\tilde{R})}{k_{a} k_{m}}\left[M_{E}+\right. \\
\left.+M_{d f}+\widetilde{M}_{d f}\right]+\frac{L \dot{M}_{E}}{k_{a} k_{m}},
\end{gathered}
$$

where $\alpha_{l}^{d}$ is a desired angle of rotation of the actuator gear output shaft; $F(p, \varepsilon)$ is a function, which describes a typical used regulator $R, p$ is a differential operator; $M_{d f}=M_{d f 0} \operatorname{sign}(\dot{\alpha}), \widetilde{M}_{d f}=\widetilde{M}_{d f 0} \operatorname{sign}(\dot{\alpha})$.

The analysis of components of law (13) shows that the synthesis of proposed faults accommodation system for each actuator of MM is no represented the difficult. Block scheme of actuator with faults accommodation system (13) is shown in Fig. 1. This system can be realized by using the typical microcontrollers.

In such a way, it is possible to provide accurate stabilization of the dynamic properties of the actuators of multilink manipulators, which are described by 
complicated nonlinear differential equations with variable parameters, by using additional signals of residuals in the law of self-tuning, even in the presence of faults.

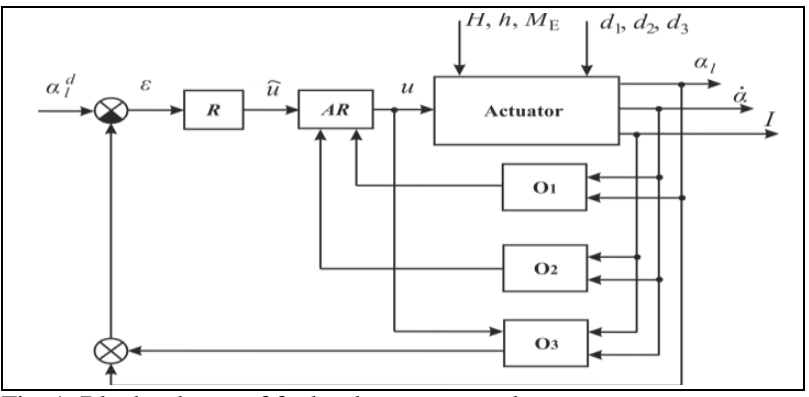

Fig. 1. Block scheme of fault tolerance control system

\section{SIMULATION OF THE SYNTHESIZED SYSTEM}

To verify the functionality and effectiveness of the proposed method, the modeling of the synthesized faults accommodation system for the model of the actuator of the second degree of freedom $\left(q_{2}\right)$ of PUMA-type manipulator (see Fig.2) was carried out.

In modeling the following values of parameters of MM's links were used: $m_{1}=35.8 \mathrm{~kg}, m_{2}=3.5 \mathrm{~kg}, m_{3}=3 \mathrm{~kg}$, $l_{1}=0.2 \mathrm{~m}, l_{2}=0.5 \mathrm{~m}, l_{3}=0.4 \mathrm{~m}, l_{1}^{*}=0.1 \mathrm{~m}, l_{2}^{*}=0.12 \mathrm{~m}, l_{3}^{*}=0.1 \mathrm{~m}$, $J_{s 2}=0.0047 \mathrm{~kg} \cdot \mathrm{m}^{2}, J_{n 2}=0.12 \mathrm{~kg} \cdot \mathrm{m}^{2}, J_{n 2}=0.067 \mathrm{~kg} \cdot \mathrm{m}^{2}, m_{\mathrm{g}}=0.5 \mathrm{~kg}$.

At the synthesis of faults accommodation system were used the following parameters of actuator with DC motor: $J=0.0001 \mathrm{~kg} \cdot \mathrm{m} \cdot \mathrm{s}^{2}, \quad k_{\omega}=0.02 \mathrm{~V} \cdot \mathrm{s}, \quad k_{a}=100, \quad R=0.4 \Omega$, $L=0.004 \mathrm{Hr}, \quad k_{m}=0.02 \mathrm{~N} \cdot \mathrm{m} / \mathrm{A}, \quad \mu=100, \quad k_{v}=0.005 \mathrm{~N} \cdot \mathrm{m} \cdot \mathrm{s} / \mathrm{rad}$, $M_{d f 0}=0.06 \mathrm{~N} \cdot \mathrm{m}$.

On the DC motor were submitted the following input signals: $\alpha_{l}^{d}=\sin (0.5 t), q_{1}=\sin (t), q_{3}=\sin (0.7 t+0.1)$. Faults were simulated by abrupt increase the value of active resistance by $50 \%$ at time $t_{1}(\tilde{R}=0.2 \Omega)$, the value of dry friction by $50 \%$ at time $t_{2} \quad\left(\widetilde{M}_{d f 0}=0.03 \mathrm{~N} \cdot \mathrm{m}\right)$ and introduction of a constant error in the position sensor of gear output shaft at the moment $t_{3}\left(\tilde{\alpha}_{l}=0.005 \mathrm{rad}\right)$.

The errors of position of actuator are shown on Fig.3.

On this figure, the error of actuator with the appearance of faults without introducing of proposed in this paper fault tolerance control system (see curve 1), and with the introduction of this system (see curve 2) are presented.

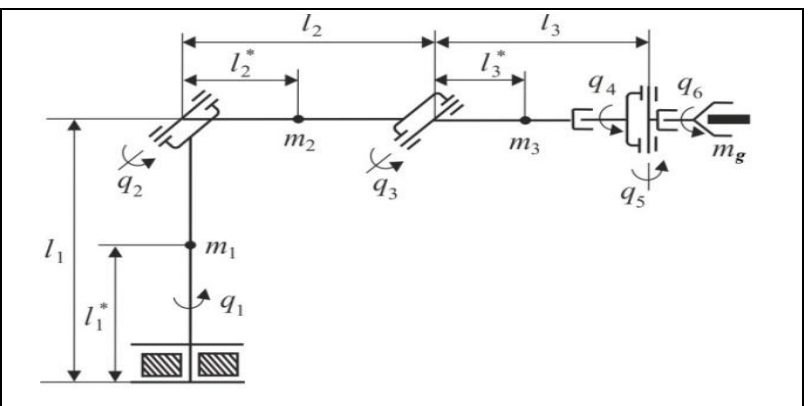

Fig. 2. Kinematical scheme of the manipulator

The Fig. 3 shows that the negative effect of these faults on the accuracy of the considered actuator is fully excluded, due to the proposed fault tolerance system.

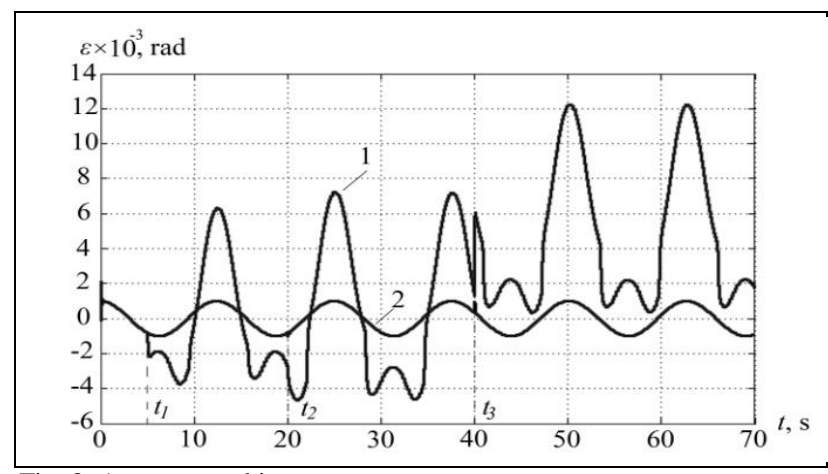

Fig. 3. Actuator tracking errors

\section{CONCLUSION}

In this paper we propose an effective method for the synthesis of high-quality faults accommodation system for actuators with DC motors of multilink manipulators described by nonlinear differential equations with variable parameters. This system provide the invariance dynamic properties of actuators to faults due to a specially formed additional control signals for actuators, that guarantees serviceability and effectiveness of manipulators during operation. Future researches will be dedicated to development of accommodation systems to faults in sensors of underwater vehicles and mobile robots on the basis of this method.

This paper is supported by RFBR (Grants \#11-0898505 and \#11-07-98502)

\section{REFERENCES}

[1]. Blanke, M.; Kinnaert, M.; Lunze J. \& Staroswiecki, M. (2003). Diagnosis and Fault Tolerant Control, Springer-Verlag, Berlin

[2]. Mironovsky, L. (1998). Functional diagnosis of dynamic systems, MSU-GRIF, St. Petersburg, Russia

[3]. Isermann, R. (2006). Fault-Diagnosis Systems: An Introduction from Fault Detection to Fault Tolerance, Springer-Verlag, Berlin

[4]. Shumsky, A.Ye.; Zhirabok, A.N. (2006). Nonlinear diagnostic filter design: Algebraic and geo-metric points of view, Int. J. Applied Mathematics and Computer Science, Vol. 16, No. 1, 2006, pp. 115-127

[5]. Frank, P. (1990). Fault diagnosis in dynamic systems using analytical and knowledge-based re-dundancy - A survey and some new results, Automatica, Vol. 26, 1990, pp. 459-474

[6]. Staroswiecki, M.; Yang, H. \& Jiang, B. (2006). "Progressive accommodation of aircraft actuator faults", Proc. IFAC Symp. Safeprocess, Beijing, China, pp. 877-882

[7]. Siqueira, A.; Terra, M. (2004). A Fault Tolerant Manipulator Robot Based on $\mathrm{H}_{2}, \mathrm{H}_{\infty}$ and Mixed $\mathrm{H}_{2} / \mathrm{H}_{\infty}$ Markovian Controls, Proc. of the IEEE International Conference on Control Applications, Taipei, Taiwan, pp. 309-314

[8]. Staroswiecki, M. (2005). Fault tolerant control: the pseudo-inverse method revisited, Proc. 16th IFAC Congr, Prague, Czech

[9]. Jang, B.; Staroswiecki, M. \& Cocquempot, V. (2003). Active fault tolerant control for a class nonlinear systems, Proc. IFAC Symp. Safeprocess, Washington, USA, pp. 127-132

[10]. Shumsky, A.; Zhirabok, A.; Jiang, B.; Zhang K. (2009). Fault accommodation in dynamic systems: fault decoupling based approach, Proc. IEEE Conference CDC, Shanghai, China, pp. 8464-8469

[11].Zhirabok, A. \& Usoltsev, S. (2001). Linear methods for fault diagnosis in nonlinear systems, Proc. European Control Conf. ECC'01, Porto, Portugal, pp. 1363-1366

[12].Filaretov, V.F. (2007). Synthesis of Adaptive Control Systems for Electric Servo Actuators of Manipulators, Proc. of the 18th DAAAM Int. Symp. "Intelligent Manufacturing \& Automation», Zadar, Croatia, 2007, pp. 277- 279 